\title{
Influence of index matching on AA/PVA photopolymers for low spatial frequency recording
}

\author{
R. Fernández, ${ }^{1}$ S. Gallego, ${ }^{1,2, *}$ A. Márquez, ${ }^{1,2}$ J. Francés, ${ }^{1,2}$ \\ F. J. Marínez, ${ }^{1}$ and A. Beléndez ${ }^{1,2}$ \\ ${ }^{1}$ Instituto Universitario de Física Aplicada a las Ciencias y las Tecnologías, Universidad de Alicante, \\ Apartado 99, 03080 Alicante, Spain \\ ${ }^{2}$ Departamento de Física, Ingeniería de Sistemas y Teoría de la Señal, Universidad de Alicante, \\ Apartado 99, E03080 Alicante, Spain \\ ${ }^{*}$ Corresponding author: sergi.gallego@ua.es
}

Received 20 November 2014; revised 4 February 2015; accepted 7 March 2015; posted 9 March 2015 (Doc. ID 227042); published 3 April 2015

\begin{abstract}
Photopolymers present appealing optical properties for holographic and diffractive applications. They enable modulation of the electrical permittivity and thickness and are self-processing, and layers with a wide range of thicknesses and properties can be fabricated on demand. In order to obtain a complete characterization of the material, low spatial frequency analysis has become a fundamental tool because the motion of the components inside of the material can be measured. We propose to use an index matching component to carry out a complete characterization and to differentiate the "apparent" and the real monomer diffusion. We also have quantified the minimum thickness to obtain the phase modulation of $2 \pi$ required for the fabrication of many diffractive elements such as lenses, axicons, or blazed gratings. Finally, we have studied the influence of the thermal effects in the thickness variations. (c) 2015 Optical Society of America

OCIS codes: (210.0210) Optical data storage; (160.0160) Materials; (170.3660) Light propagation in tissues; (090.0090) Holography; (090.1970) Diffractive optics.

http://dx.doi.org/10.1364/AO.54.003132
\end{abstract}

\section{Introduction}

Photopolymers are among the most promising holographic recording media for many technological applications, from integrated optical waveguide fabrication to optical data storage [1-5] . Many families of photopolymers have been designed in order to emphasize some aspects of the material. For example, some compositions present particularly low scattering, high refractive index modulation, high thickness, environmental compatibility, or high or low monomer diffusion [6-11].

The recording of very low spatial frequencies on photopolymers permits us to measure the diffusion

$1559-128 X / 15 / 113132-09 \$ 15.00 / 0$

(C) 2015 Optical Society of America effects in real time, and also it is interesting to explore the fabrication of diffractive optical elements (DOEs) using these materials [12-14]. In general to record a DOE on photopolymers, an amplitude modulated beam is projected onto the material producing changes in the thickness and in the refractive index. As a result a phase diffracted element is stored. The material response depends on many parameters. When all of them are accurately determined, the material behavior can be modeled [15-20], enabling us to easily find the optimum schedule to multiplex many holograms for holographic memories [21,22], or the intensity distribution to obtain the desired DOE with the required relief structure or refractive index distribution [14]. To manufacture DOEs we have used a hybrid optical-digital system with a liquid crystal spatial 
light modulator (LC-SLM) performing the function of the master [14]. In the manufacture of DOEs we need to characterize the phase modulation properties that the photopolymer provides as a function of the exposure. To do this, novel real-time measuring techniques have been proposed, incorporating an interferometric system in the recording setup [23].

At very low spatial frequency recording, the thickness variations play an important role in DOE formation [13]. Some authors have exploited this property to generate these relief structures to confine liquid crystal molecules [24] or to fabricate relief DOEs. Nevertheless, in order to perform an accurate analysis it is important to uncouple the relief variations and refractive index changes during the recording process and the post-evolution when the material is kept in darkness. To analyze and to measure separately the thickness changes, it is possible to study the reflected beam for a wavelength at which the dye does not absorb.

On the other hand, once the DOE is stored in the material, the transmitted beam has the information of the thickness and the refractive index modulation mixed. One interesting possibility to measure the changes produced by the refractive index variation separately is the incorporation of an index matching element. Index matching techniques have been classically used for holographic recording materials [25]. In principle, to obtain an accurate index matching we have to choose a liquid with refractive index very close to the mean of the polymer one. Then, when shrinkage takes place during recording, mainly due to the polymerization, the liquid will fill up the generated grooves, minimizing the diffractive effects produced by the relief structure. Some authors have proposed the use of silica oil to index match a glass plate (microscope slide) and acrylamide photopolymers [13]. It is worthwhile to note that the presence of silica oil for index matching reduces the measured monomer diffusion more than a factor of 10. The recording process is presented in Fig. 1. In this figure we show first the shrinkage in the illuminated zones mainly due to the polymerization; the material becomes more compact. Second, after illumination, the illuminated zones swell due to the "apparent" monomer diffusion. The huge difference between the diffusion times estimated with and without index matching indicates that the changes observed on the surface (surface or "apparent" diffusion) are different from the "real" or internal diffusion. Furthermore the incorporation of a coverplate and index matching systems improves the conservation and the lifetime of the recorded DOEs [26]. In addition, the reduction of the matter transfer produced by the presence of a sealant can be used to record sharp DOEs with insignificant smoothing of the refractive index profiles.

In this paper we have focused our attention on the analysis of an acrylamide, AA, based photopolymer with and without index matching. This type of photopolymer is one of the most widely studied in

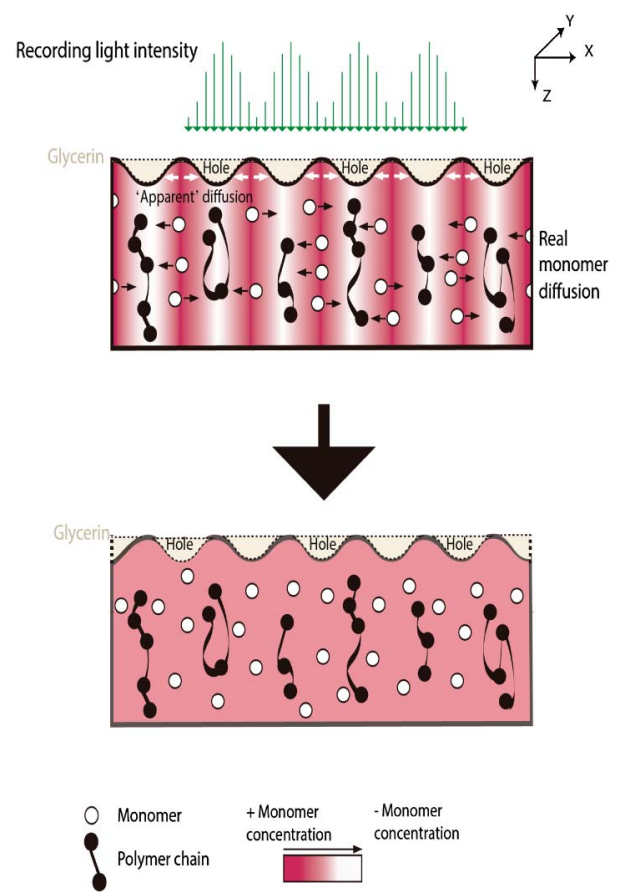

Fig. 1. Diagram of the grating recording in the photopolymers with index matching. The "apparent" diffusion is due to the recovering surface changes, and the "real" diffusion is due to the internal monomer motion.

the literature [11-14,27-29]. We want to evaluate the viability of this type of photopolymer to record DOEs and the influence of the index matching liquid. To achieve the first goal, the fabrication of lenses, axicons, or blazed gratings, a phase depth of $2 \pi$ is required. We calculate the minimum thickness to achieve the desired phase depth. In addition we study the material behavior for different spatial frequencies and calculate the "apparent" monomer diffusion for the noncovered photopolymer and the real monomer diffusion for index matched samples, which will be conveniently defined in Section 3 .

\section{Experimental Setup}

As we have said in Section 1, one of the most classical photopolymers is compose $\bar{d}$ of AA as a polymerizable monomer, triethanolamine (TEA) as a coinitiator and plasticizer, yellowish eosin (YE) as a dye, polyvinyl alcohol (PVA) as a binder, and a small proportion of water as an additional plasticizer. It may also contain $\mathrm{N}, \mathrm{N}^{\prime}$-methylene-bis-acrylamide (BMA) as a crosslinking monomer. Different types of PVA can be used as a binder; they are classified according to their molecular weight and degree of hydrolysis, which are the factors that determine the viscosity of the prepared solutions and the polymers' capacity to retain water. The molecular weight range is between 20,000 and 200,000. PVA with a low average molecular weight has short chains and produces solutions of low viscosity and little capacity to retain water per unit of mass. The remaining water in the material has a direct influence both on the 
Table 1. Composition of the Liquid Solution for Photopolymer AA

\begin{tabular}{lccc}
\hline TEA $(\mathrm{ml})$ & PVA $(\mathrm{ml})(8 \% \mathrm{w} / \mathrm{v})$ & $\mathrm{AA}(\mathrm{gr})$ & $\mathrm{YE}(0.8 \% \mathrm{w} / \mathrm{v})(\mathrm{ml})$ \\
\hline 2.0 & 25 & 0.96 & 0.6 \\
\hline
\end{tabular}

material diffusion, as shown in Ref. [30], and on the size of PVA molecules in the material resolution, as demonstrated by Zhu et al. [27]. In this work we have used a PVA 18-88 with $\mathrm{Mw}=180,000$ amu in order to obtain the highest limit for the species diffusion inside the layer. Besides, for low spatial frequency recording we do not need to achieve high resolution. The particular concentration used in this work is presented in Table 1 . To analyze the simplest chemical composition we avoid the presence of a crosslinker to study the material variations after recording with and without index matching and coverplate. To index match the photopolymer we used a glycerin with refractive index $n=1.478$. This value fits perfectly with our pre-exposed photopolymer with a refractive index of 1.477 measured with a refractometer and published in previous works such as Ref. [31]. In addition it is possible, as recommended in Ref. [32], to use commercial baby oil; we found that it is mainly composed of glycerin, with refractive index of 1.484 that provides results similar to pure glycerin.

$30 \mathrm{ml}$ of solution with water as the solvent is deposited, using the force of gravity, on a glass substrate $(25 \mathrm{~cm} \times 20 \mathrm{~cm})$, and left in the dark $\left(\mathrm{RH}=40 \%-45 \%, T=20-23^{\circ} \mathrm{C}\right)$. When part of the water has evaporated (after about $36 \mathrm{~h}$ ), the layer has enough mechanical resistance and can be cut without deforming. The final "solid" film has a physical thickness around $90 \pm 5 \mu \mathrm{m}$. This final thickness can be modified, changing the quantity of the syrup deposited on the glass. More specifically, layers with a thickness between 30 and $250 \mu \mathrm{m}$ using the same method of preparation have been successfully fabricated [33].

To store DOEs onto photopolymers and also for measuring the velocity of monomer diffusion, the setup presented in Fig. 2 is considered. In order to record phase diffractive gratings we introduced a spatial light modulator (SLM) working in the amplitude-only mode to modulate the green beam $(532 \mathrm{~nm})$; the materials described in this work are

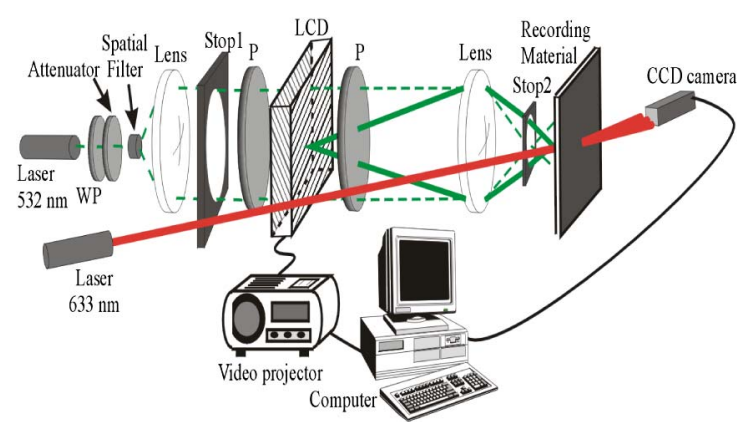

Fig. 2. Experimental setup to record sinusoidal gratings onto the photopolymer using the LCD as a master. sensitized to green light and transparent for red light $(633 \mathrm{~nm})$. The periodic pattern, sinusoidal-like, is introduced by a liquid crystal display (LCD), a Sony LCD model LCX012BL, extracted from a video projector, Sony VPL-V500. We use the electronics of the video projector to send the voltage to the pixels of the LCD. The LCD is used in the amplitude-mostly modulation regime by proper orientation of the external polarizers $(\mathrm{P})$; then the pattern is imaged onto the material with an increased spatial frequency (a demagnifying factor of 2). The use of the LCD allows us to change the period of the grating recorded in the photopolymer without moving any mechanical part of the setup. Nevertheless the size of the pixel, $42 \mu \mathrm{m}$, of this LCD model together with the filtering of the pixelation of the LCD (by stop 2) limits the minimum value of the spatial period in the recording material to $168 \mu \mathrm{m}$ (i.e., $8 \mathrm{LCD}$ pixels to reproduce a period).

\section{Results and Discussion}

In this section we present the results obtained for some of the samples analyzed. Moreover, the behavior after illumination is studied, providing interesting information about the internal mechanism and the molecules' migration. In order to calculate the phase depth achieved in the recording of a thin phase sinusoidal diffraction grating, we can compare the diffraction efficiencies (DEs) of the main diffracted orders to the theoretical ones in the Fraunhofer domain given by the Bessel equations [32]. In Fig. 3 we present the behavior of the four main diffraction orders for a thin sinusoidal phase grating as a function of the phase depth. The comparison of our diffraction efficiency results with this figure provides us a value of phase depth achieved for each material, and we compare it to the value of $2 \pi$, required for many interesting diffractive applications, so we can estimate the necessary thickness to achieve this value for each particular chemical composition. We proved in previous works that for this range of spatial frequencies the behavior of AA photopolymers is almost linear [15].

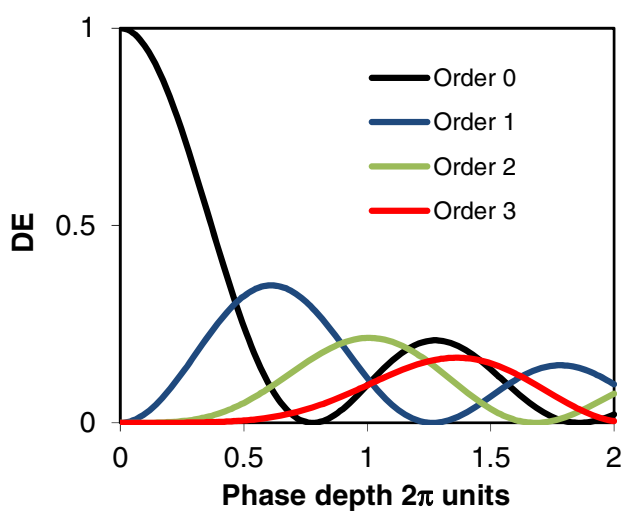

Fig. 3. Diffraction efficiency for the first four orders of a sinusoidal grating as a function of the phase depth. 


\section{A. Effect of Index Matching}

The diffraction efficiency at very low spatial frequency ranges depends on two aspects: the surface changes and the refractive index distribution inside the material. To avoid the influence of the relief structures formed, Close et al. proposed index matching using silica oil and coverplating the sample with microscope slides [13]. Therefore the diffracted intensities, in this case, depend only on the refractive index distribution produced by the polymerization. The diffraction efficiencies of the main diffracted orders as a function of time can be seen in Figs. 4(a) and $\underline{4(\mathrm{~b})}$, uncoverplated and coverplated, respectively; phase modulation for longer time exposures (>300 s) of the grating looks very similar in both cases. As mentioned in Fig. 4(a), uncoverplated, the recorded phase gratings can be understood as the superposition of two phase gratings: a relief grating plus a refractive index grating. Since the final DEs are very similar after $300 \mathrm{~s}$, it can be assumed that the effect of the relief grating is weak after this long time. This result makes sense if we take into account the fast values of the surface recovering velocities or the monomer diffusion through the surface measured by many authors [12,34]: in general the values of the "apparent" diffusion coefficients
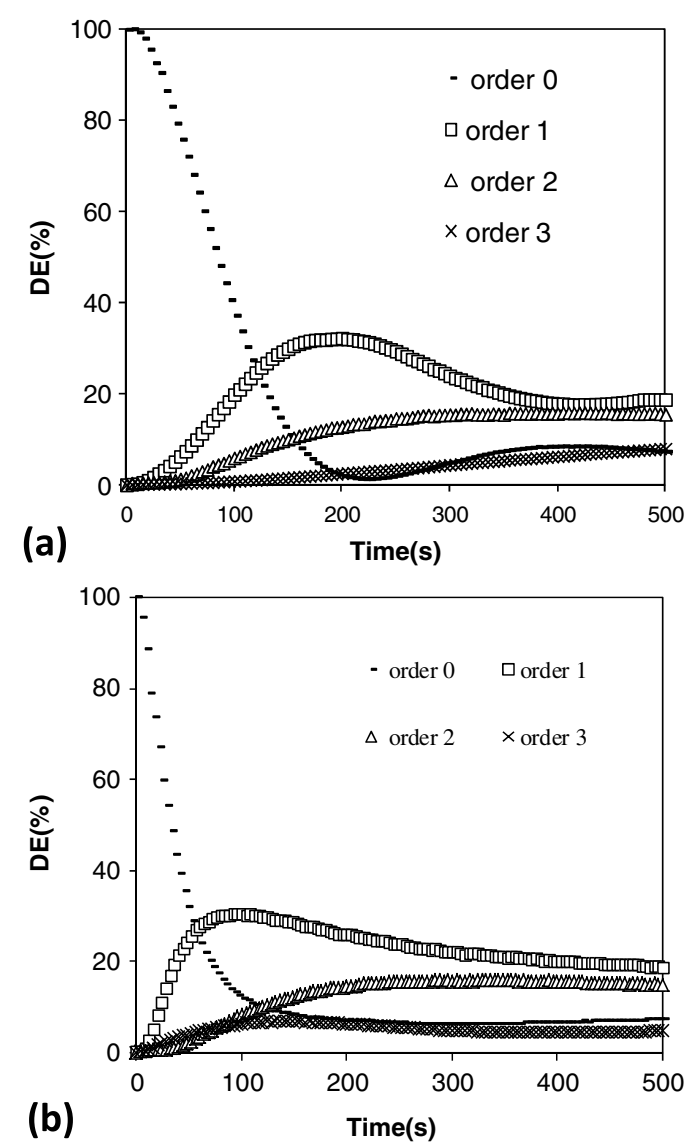

Fig. 4. Experimental diffraction efficiencies during recording of a grating with spatial period of $168 \mu \mathrm{m}$ and thickness of $85 \mu \mathrm{m}$ (a) without coverplating and (b) index matched and coverplated. are around $10^{-8} \mathrm{~cm}^{2} / \mathrm{s}$. One additional effect due to the relief grating observed in Fig. 4(a) is that the maximum of the order 1 is achieved at $180 \mathrm{~s}$, three times later than in Fig. 4(b), 60 s. This happens because of the shrinkage produced in the illuminated zones: the shrinkage produces a decrease in the phase modulation quickly corrected at longer exposure times by the fast diffusion through the surface. Furthermore, comparing these figures with Fig. 2 , it can be deduced that the phase depth achieved is around $1.7 \pi$ after $500 \mathrm{~s}$ of recording in both cases. This value is not enough to record useful diffractive elements such as lenses, axicons, or blazed gratings. The physical thickness of these layers was measured using a ultrasound display provided by Neurtek, and the value obtained was $85 \pm 3 \mu \mathrm{m}$; after that we have recorded a holographic grating to confirm this value fitting the angular response around Bragg's angle. There are many ways to increase the phase amplitude of the grating. Two of the most studied are including crosslinker monomers or increasing the thickness of the sample. The first one has been studied by many authors $[28,29]$, and the results of the second one are presented in Fig. 5 , where orders 0 and 1 are depicted as a function of time for a grating $105 \pm 3 \mu \mathrm{m}$ thick. From the analysis of Fig. $\underline{2}$ it can be identified that the phase modulation is higher than $2 \pi$.

\section{B. Different Spatial Periods}

In order to store more complex recording elements in the photopolymer, it is interesting to analyze the material response for different spatial frequencies. In previous work [37] it can be seen that for noncoverplated layers the photopolymer presents a different behavior when we change the recorded spatial period from 168 to $672 \mu \mathrm{m}$. This can be explained by the strong influence of the relief gratings formed due to the shrinkage produced in the illuminated areas. The time diffusion constant, $\tau_{D}$, is usually defined as follows [30]:

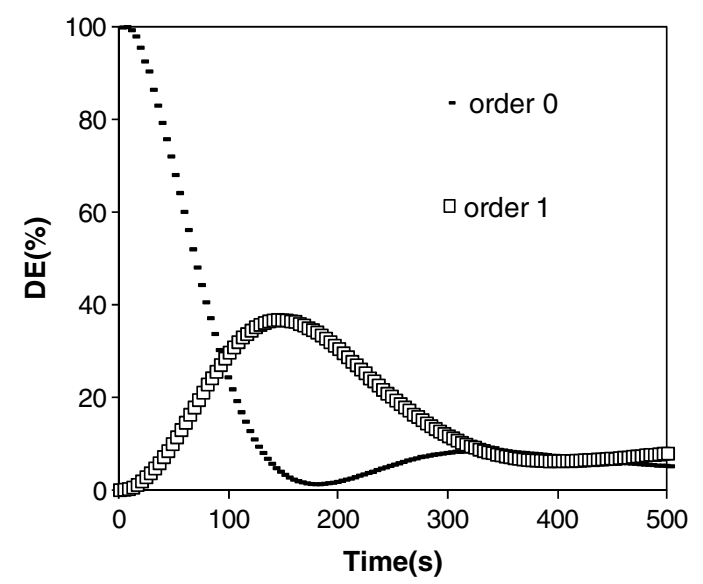

Fig. 5. Experimental diffraction efficiencies during recording of a grating with spatial period of 168 and $105 \mu \mathrm{m}$ thickness without coverplating. 


$$
\tau_{D}=\frac{1}{K_{g}^{2} D},
$$

where $K_{g}$ is the grating number and $D$ is the diffusion coefficient. $\tau_{D}$ shows the average time for a particle to travel one period. When the value of $\tau_{D}$ is comparable to the recording time, this diffusion has an influence on the material behavior. The surface recovering times measured by many authors $[12,13,15]$ indicate that the diffusion in the relief gratings becomes significant for the period of $168 \mu \mathrm{m}$ in opposition to $672 \mu \mathrm{m}$. In the latter case, this influence is exhibited after recording but not during the polymerization process. Therefore, this behavior of the material across this spatial period is also expected, since neglecting the influence of the relief on DEs is avoided. Figures $6(\mathrm{a})$ and $6(\mathrm{~b})$ present the results for 336 and $672 \mu \mathrm{m}$, respectively. It can be seen that the behavior is very similar for the three spatial frequencies analyzed. It seems clear that the monomer has very weak influence on the DOE fabrication. This means that the timescale of diffusion across a fringe is well separated from the timescale of polymerization, so that the material response is uniform,
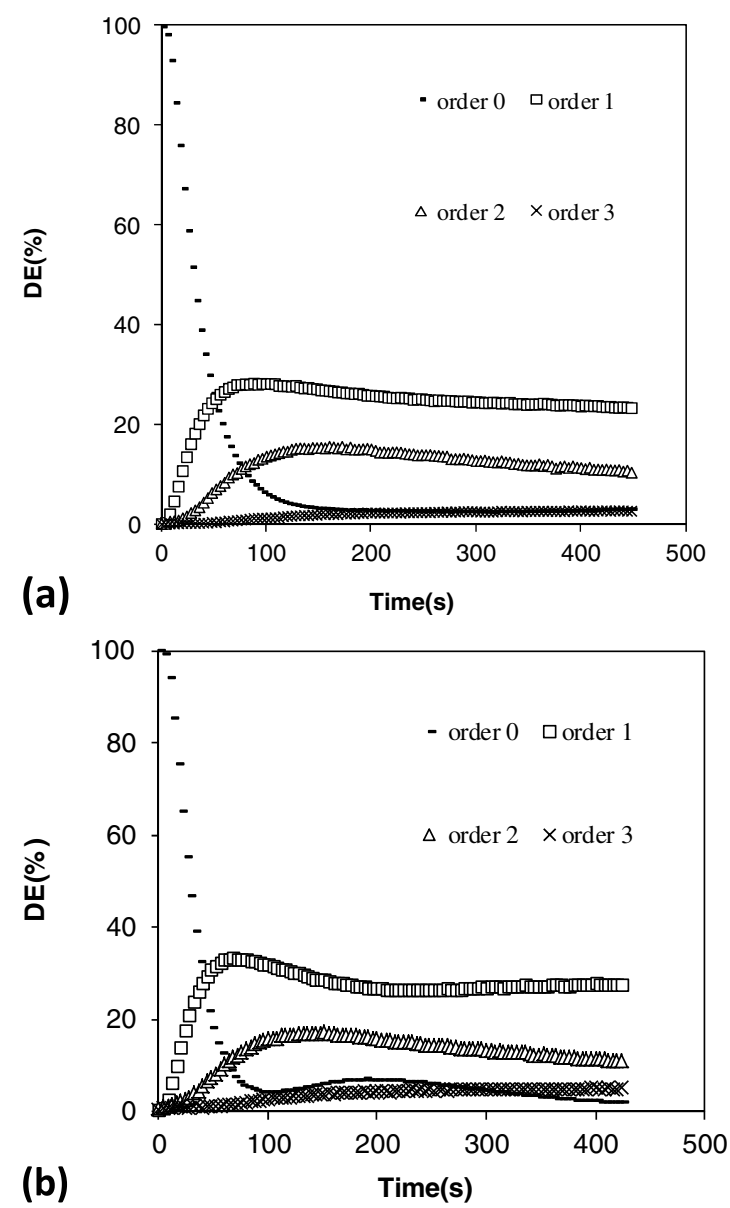

Fig. 6. Experimental diffraction efficiencies during recording for matched samples with thickness of $85 \mu \mathrm{m}$ and with spatial period (a) $336 \mu \mathrm{m}$ and (b) $772 \mu \mathrm{m}$. as desired, over the examined range of spatial frequencies. This evidence suggests that for manufacturing of more complex DOEs such as diffractive lenses, composed by many spatial frequencies, we can expect similar behavior for each particular frequency. Therefore, good agreement between the phase element stored and the intensity distribution projected on the material is obtained. This situation is ensured whenever short recording times are used, less than $80 \mathrm{~s}$, since it avoids saturation of the material.

\section{Post-Exposure Evolution}

In the usual optical holographic regime (high spatial frequencies), the diffusion times are very short, less than $0.1 \mathrm{~s}$, and it is very difficult to measure them. Therefore, for spatial frequencies around 1000 lines/mm, species diffusion can only be determined indirectly. For very low spatial frequency recording we can measure more than eight diffracted orders and obtain reliable information about the phase profile recorded in the material and its evolution after recording due to diffusion.

After measuring the diffracted efficiency of the main orders as a function of time we can use the Fermi-Dirac function to fit the shape of the recorded profile [35]. Nevertheless in this paper we assume that the phase modulation profile $\varphi(x)$ is close to the sinusoidal profile; thus we can calculate the phase modulation comparing the refractive index of both the zeroth and first orders to the values provided in Fig. 3.

In this sense, to solve Fick's equation [see Eq. (2)] after a periodic illumination we proposed the following model. Due to exposure a species concentration distribution is generated inside the photopolymer $\left(\phi^{(i)}\right)$. Exponential decay (increase) of the species concentration in the nonexposed (exposed) zones due to species diffusion begins when exposure stops to achieve the average value of the residual species concentration $\left(\phi_{f}^{(i)}\right)$, corresponding to the point at which monomer diffusion eventually stops due to a uniform monomer distribution. Therefore, using this method we obtain the average apparent diffusion value, $D$, for all the molecules, $\phi^{(\text {avg })}$. It is worthwhile to note that we call $D$ "apparent" diffusion so as to differentiate the surface and internal changes. In the next equation we show Fick's equations:

$$
\frac{\partial \phi^{(i)}(x, t)}{\partial t}=\frac{\partial}{\partial x}\left[D(x, t) \frac{\partial \phi^{(i)}(x, t)}{\partial x}\right] .
$$

We can thus describe the species concentration after exposure as

$$
\phi^{(i)}(x, t)=\phi_{f}^{(i)}+\Delta \phi^{(i)}(x) \exp \left(\frac{-t}{\tau}\right),
$$

where the species modulation is $\Delta \phi^{(i)}(x)$ and $\tau$ is the characteristic time of monomer variation and can be calculated by fitting the phase depth of the grating 
variation with time [14]. When we introduce this expression in Eq. (2) we obtain

$$
\Delta \phi^{(i)}(x, t)=-\tau \frac{\partial}{\partial x}\left[D(x, t) \frac{d \Delta \phi^{(i)}(x)}{d x}\right] .
$$

In a first approximation we can assume a sinusoidal distribution of the modulation of species concentrations and for the illumination $(I)$,

$$
\begin{gathered}
\Delta \phi^{(i)}(x)=\Delta \phi_{0}^{(i)}(x) \cos \left(\frac{2 \pi x}{\Lambda}\right), \\
I(x)=I_{0}\left(1-\cos \left(\frac{2 \pi x}{\Lambda}\right)\right),
\end{gathered}
$$

where $\Lambda$ is the grating period. This assumption only makes sense for short exposures, without saturation, and sinusoidal distribution of the recording intensity [15]. Therefore Eq. (ㅁ) can be rewritten as

$$
\operatorname{tg}\left(\frac{2 \pi}{\Lambda}\right) \frac{\partial}{\partial x} D(x, t)+\frac{2 \pi}{\Lambda} D(x, t)-\frac{1}{2 \pi \tau}=0
$$

This gives a first-order inhomogeneous differential equation with constant coefficients; therefore, the general solution is

$$
D(x)=\frac{\Lambda^{2}}{4 \pi^{2} \tau}+D_{1} \csc \left(\frac{2 \pi}{\Lambda} x\right) .
$$

It is worth noting the simplicity of Eq. (8) - only two parameters determine the value of $\mathrm{D}: \bar{\Lambda}$ and $\tau$. For short expositions we can assume that the polymer concentration is small and we can assume that $D$ is spatially independent:

$$
D=\frac{\Lambda^{2}}{4 \pi^{2} \tau} .
$$

In our analysis of the evolution of the refractive index distribution in time, we noted that the phase depth, $\Delta \varphi$, becomes time independent after a time range of several minutes to a few days after exposure. The approximation of this value is exponential. Next we show how we can obtain the value for the parameter $\tau$, needed in Eq. (9)), from the fitting of the temporal variation for $\Delta \varphi \varphi$. If we use Eq. (므) we can obtain

$$
\Delta \varphi(x, t)=V_{m} \Delta \phi^{(\mathrm{avg})}(x)\left(\exp \left(\frac{-t}{\tau}\right)-1\right),
$$

where $V_{m}$ is the molar volume of the monomer. This value tends asymptotically (in practice in some minutes) to the value

$$
\Delta \varphi(x, t \rightarrow \infty)=-V_{m} \Delta \phi^{(\mathrm{avg})}(x) .
$$

Thus, Eq. (13) can be rewritten as

$$
\Delta \varphi(x, t \rightarrow \infty)-\Delta \varphi(x, t)=\Delta \varphi(x, t \rightarrow \infty) \exp (-t / \tau) .
$$

If we apply the logarithm to both sides of the expression, we obtain

$$
\ln (\Delta \varphi(x, t \rightarrow \infty)-\Delta \varphi(x, t))=\ln (\Delta \varphi(x, t \rightarrow \infty))-t / \tau
$$

It should be noted that we did not assume any specific profile for $\Delta \varphi(x)$ to derive Eq. (8). The only assumption is that $\Delta \varphi$ is proportional to $\Delta \phi^{\text {(avg) }}$, which is reasonable for small polymer concentrations. We have presented the fast post-recording evolution of the DEs for layers without index matching and with index matching in Fig. $\underline{7}$. It can be seen that the variation of the DEs for uncovered layers is very fast in comparison to that for the index matched ones. It should be remarked that very small variations in the thickness of the layer, less than $2 \mu \mathrm{m}$, produce significant changes in the diffracted energy. On the other hand, in the index matched case, variations due to swelling or shrinkage are avoided and only the migration of molecules inside the material affects the changes in the DEs. Therefore, in Fig. 7, for cover layers, we can observe that after recording, the $\mathrm{DE}$ of the two main orders remains practically constant. In fact, we have to wait several minutes to measure the tendency in the post-recording evolution.

Following the method described in Eqs. (7)-(10), the value of the apparent monomer diffusion through the surface for uncovered samples can be fitted. In addition, using data provided in Fig. $\underline{7}$ the diffusion inside the material, the real monomer diffusion, or bulk diffusion in the index matched samples can be fitted. The results are depicted in Figs. 8(a) and $\underline{8(b)}$ for uncovered and index matched, respectively.

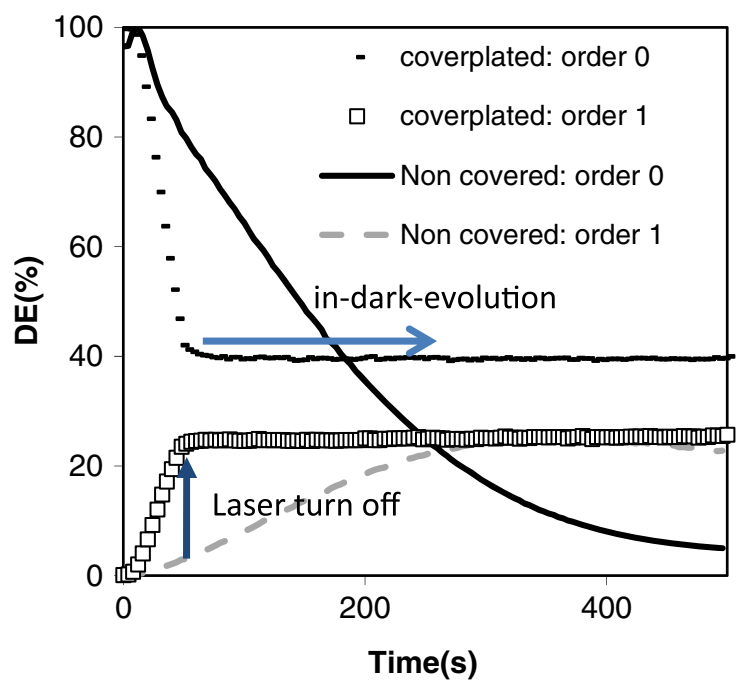

Fig. 7. Experimental diffraction efficiencies for chemical composition AA1 during $50 \mathrm{~s}$ recording of a grating with spatial period $168 \mu \mathrm{m}$ and the post-evolution, a sample with $85 \mu \mathrm{m}$ thickness without coverplating and index matched and coverplated. 

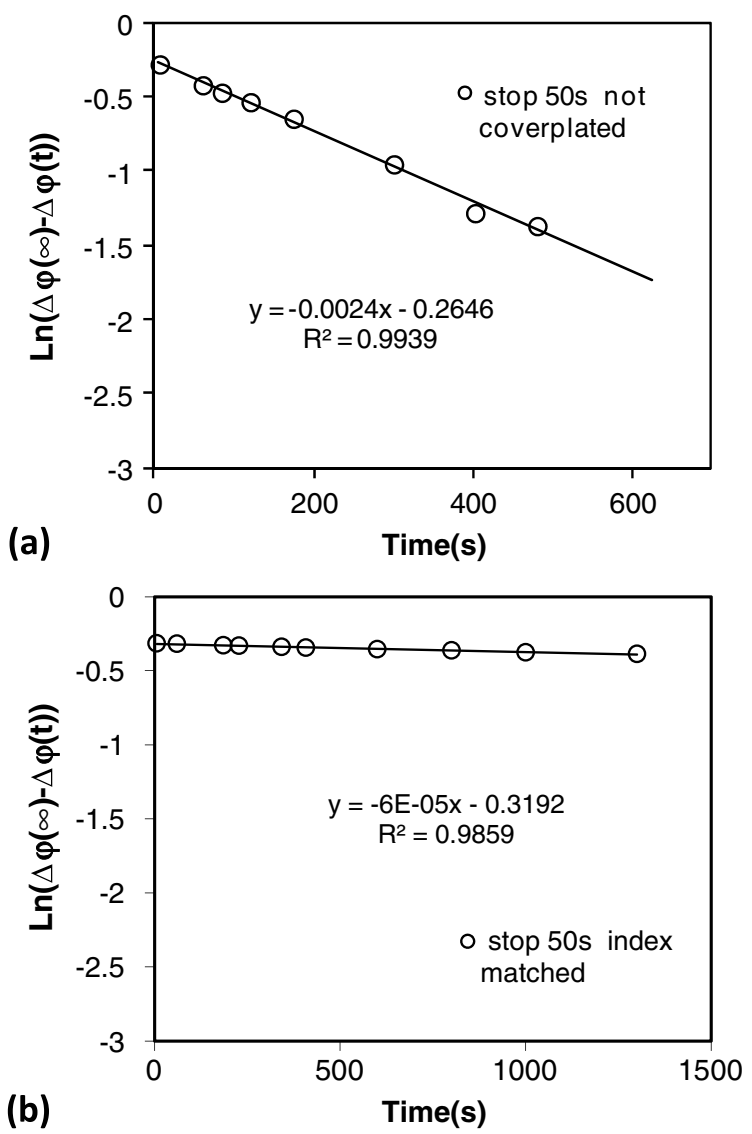

Fig. 8. Experimental values fitted using Eq. (13) after the $50 \mathrm{~s}$ of recording for composition AA1; grating with spatial period of $168 \mu \mathrm{m}$ (a) without coverplating and (b) index matched and coverplated.

It can be identified that the slope of the linear function is almost two magnitude orders higher for uncovered layers. Thus the value obtained for "apparent" diffusivity for uncovered layers is $1.6 \cdot 10^{-8} \mathrm{~cm}^{2} / \mathrm{s}$, and the value for the monomer diffusion for index matched is $3.5 \cdot 10^{-10} \mathrm{~cm}^{2} / \mathrm{s}$. This second value of monomer diffusion agrees with the expected magnitude of the secondary harmonics in the refractive index profile measured in registration of holographic sinusoidal gratings [36]. It is worthwhile to note that the error measured in the coverplated case is clearly higher than that obtained in the uncovered case, due to the very slow variation of the $\mathrm{DE}$ and the the calculation of $\Delta \varphi(x, t \rightarrow \infty)$. In our experiments we have found values for the monomer diffusion in the range from $4 \cdot 10^{-10}$ to $10^{-10} \mathrm{~cm}^{2} / \mathrm{s}$. Recording higher values of spatial frequencies, this error can be reduced due to the long distances traveled by the monomer; nevertheless the time extension required to obtain $\Delta \varphi(x, t \rightarrow \infty)$ is also increased. Finally, it is important to remark that the apparent diffusion is more or less constant for different spatial frequencies, as has recently been presented in [37]. Nevertheless we agree with the remarks postulated by Close et al. in [13]: "in general, as the spatial period increases, the predicted rates of apparent/surface diffusion also increase. This can be

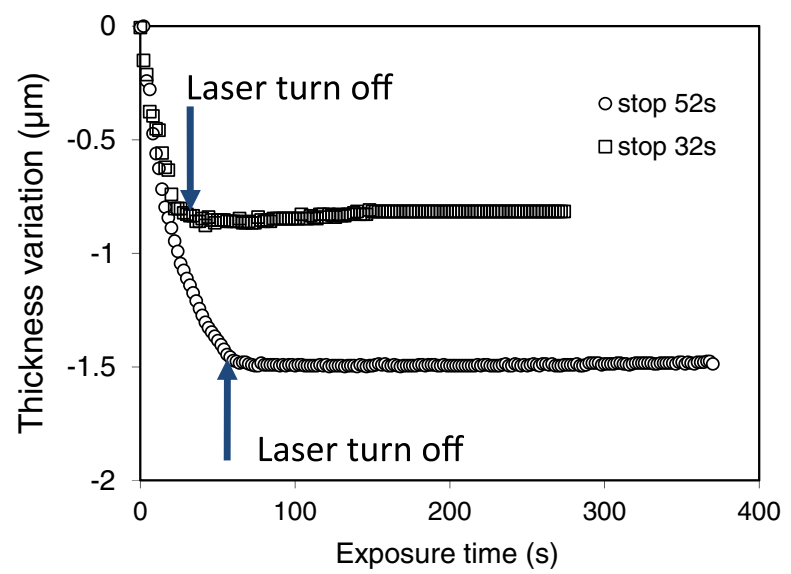

Fig. 9. Shrinkage produced during $52 \mathrm{~s}$ of uniform exposure and the post-evolution for a photopolymer without coverplating for a layer with $89 \mu \mathrm{m}$ of physical thickness.

explained in terms of the effect of surface tension on the formation of the surface profile. For larger periods, the height variations are larger and take place more rapidly due to the lower energy restriction imposed by the lower surface curvatures in these profiles."

To be sure that the changes in the photopolymer relief are not originated by thermal effects, we develop a straightforward experiment. Using interferometric methods it is possible to measure in real time the shrinkage produced during polymerization [23]. In this case we use a uniform green beam, $532 \mathrm{~nm}$, with an intensity of $0.5 \mathrm{~mW} / \mathrm{cm}^{2}$ and with a circular section with a radius of $1 \mathrm{~cm}$, as in the experiments carried out in Ref. [23]. After 32 and $52 \mathrm{~s}$ of uniform exposure, we present two different experiments; the green laser is turned off, and then if some thermal effects are important, due to the exothermal polymerization reaction, some water is evaporated and the material shrinks, and thus we would expect a recovery in thickness. Furthermore, if the photopolymer is cooling down, an exponential variation of the thickness should be measured. Figure 9 shows evidence that the shrinkage is practically constant immediately after the illumination for both experiments. Note that a very weak increase in shrinkage can be measured. Therefore we can assume that the thermal effects do not drastically affect the material thickness for very low spatial DOE recording.

\section{Conclusions}

The recording of low spatial frequency in photopolymers has been analyzed in this paper. In particular, a deep study has been carried out regarding the inclusion of a microscope slide as a coverplate with a transparent liquid to index match in PVA/AA-based materials. It has been shown that the fast changes measured in uncovered materials, $D \sim 10^{-8} \mathrm{~cm}^{2} / \mathrm{s}$, are due to the changes produced in the surface. Moreover, the values measured in coverplated samples, $D \sim 10^{-10} \mathrm{~cm}^{2} / \mathrm{s}$, are close to the "classic" ones proposed for photopolymers, and that indicates the 
very weak influence of monomer diffusion in the DOE fabrication, as has been shown by the similar behavior for three different spatial periods. In any case, for covered layers and to minimize the error in the diffusion constant fittings, the experiments designed have included the recording of higher spatial frequencies or large post-exposure times. Finally we have reported experimental evidence to demonstrate that the thermal effects do not seem to be affected during recording. Further experiments and hypotheses can be proposed to explain the changes in the material relief. The results presented show that in the case of PVA materials a phase depth of $2 \pi$ can be achieved with samples with thickness of $105 \mu \mathrm{m}$ even without the inclusion of crosslinked monomers in the chemical composition.

The work was supported by the "Ministerio de Economía y Competitividad" of Spain under projects FIS2011-29803-C02-01 and FIS2011-29803-C02-02 and by the "Generalitat Valenciana" of Spain under projects PROMETEO/2011/021 and ISIC/2012/013.

\section{References}

1. J. Neumann, K. S. Wieking, and D. Kip, "Direct laser writing of surface reliefs in dry, self-developing photopolymer films," Appl. Opt. 38, 5418-5421 (1999).

2. X. T. Li, A. Natansohn, and P. Rochon, "Photoinduced liquid crystal alignment based on a surface relief grating in an assembled cell," Appl. Phys. Lett. 74, 3791-3793 (1999).

3. C. P. Jisha, V. C. Kishore, B. M. John, V. C. Kuriakose, K. Porsezian, and C. Sudha Kartha, "Self-written waveguide in methylene blue sensitized poly(vinyl alcohol)/acrylamide photopolymer material," Appl. Opt. 47, 6502-6507 (2008).

4. M. D. Lechner, "Photopolymers for optical memories and waveguides," in Electronic Properties of Polymers and Related Compounds, Vol. 63 of Springer Series in Solid-State Sciences (Springer, 1985), pp. 301-308.

5. H. J. Coufal, D. Psaltis, and G. T. Sincerbox, eds. Holographic Data Storage (Springer-Verlag, 2000).

6. N. Suzuki and Y. Tomita, "Silica-nanoparticle-dispersed methacrylate photopolymers with net diffraction efficiency near 100\%," Appl. Opt. 43, 2125-2129 (2004).

7. E. Hata, K. Mitsube, K. Momose, and Y. Tomita, "Holographic nanoparticle-polymer composites based on step-growth thiolene photopolymerization," Opt. Mater. Express 1, 207-222 (2011).

8. A. Olivares-Pérez, S. Toxqui-López, and A. L. Padilla-Velasco, "Nopal cactus (Opuntia Ficus-Indica) as a holographic material," Materials 5, 2383-2402 (2012).

9. P. Cheben and M.-L. Calvo, "A photopolymerizable glass with diffraction efficiency near $100 \%$ for holographic storage," Appl. Phys. Lett. 78, 1490-1492 (2001).

10. D. Cody, I. Naydenova, and E. Mihaylova, "Effect of glycerol on a diacetone acrylamide-based holographic photopolymer material," Appl. Opt. 52, 489-494 (2013).

11. M. R. Gleeson, J. T. Sheridan, F. Bruder, T. Rölle, H. Berneth, M. Weiser, and T. Fäcke, "Comparison of a new self developing photopolymer with AA/PVA based photopolymer utilizing the NPDD model," Opt. Express 19, 26325-26342 (2011).

12. T. Babeva, I. Naydenova, S. Martin, and V. Toal, "Method for characterization of diffusion properties of photopolymerisable systems," Opt. Express 16, 8487-8497 (2008).

13. C. E. Close, M. R. Gleeson, and J. T. Sheridan, "Monomer diffusion rates in photopolymer material. Part I. Low spatial frequency holographic gratings,"J. Opt. Soc. Am. B 28, 658-666 (2011).

14. S. Gallego, A. Márquez, M. Ortuño, J. Francés, S. Marini, I. Pascual, and A. Beléndez, "Surface relief model for without coverplating photopolymers," Opt. Express 19, 10896-10906 (2011).

15. S. Gallego, A. Marquez, F. J. Martínez-Guardiola, M. Riquelme, R. Fernández, I. Pascual, and A. Beléndez, "Linearity in the response of photopolymers as optical recording media," Opt. Express 21, 10995-11008 (2013).

16. G. Zhao and P. Mouroulis, "Diffusion model of hologram formation in dry photopolymers materials," J. Mod. Opt. 41, 1929-1939 (1994).

17. J. T. Sheridan and J. R. Lawrence, "Nonlocal-response diffusion model of holographic recording in photopolymer," J. Opt. Soc. Am. A 17, 1108-1114 (2000).

18. V. Moreau, Y. Renotte, and Y. Lion, "Characterization of DuPont photopolymer: determination of kinetic parameters in a diffusion model," Appl. Opt. 41, 3427-3435 (2002).

19. G. M. Karpov, V. V. Obukhovsky, T. N. Smirnova, and V. V. Lemeshko, "Spatial transfer of matter as method of holographic recording in photoformers," Opt. Commun. 174, 391-404 (2000).

20. C. Li, L. Cao, Q. He, and G. Jin, "Holographic kinetics for mixed volume gratings in gold nanoparticles doped photopolymer," Opt. Express 22, 5017-5028 (2014).

21. J. T. Sheridan, M. G. Gleeson, J. V. Kelly, and F. T. O’Neill, "Nonlocal polymerization-driven diffusion-model-based examination of the scaling law for holographic data storage," Opt. Lett. 30, 239-241 (2005).

22. S. Gallego, M. Ortuño, C. Neipp, E. Fernández, A. Beléndez, and I. Pascual, "Improved maximum uniformity and capacity of multiple holograms recorded in absorbent photopolymers," Opt. Express 15, 9308-9319 (2007).

23. S. Gallego, A. Márquez, M. Ortuño, J. Francés, I. Pascual, and A. Beléndez, "Diffractive and interferometric methods to characterize photopolymers with liquid crystal molecules as holographic recording material," J. Eur. Opt. Soc. Rapid Pub. 7, 12024 (2012).

24. K. Pavani, I. Naydenova, S. Martin, R. Jallapuram, R. G. Howard, and V. Toal, "Electro-optical switching of liquid crystal diffraction gratings by using surface relief effect in the photopolymer," Opt. Commun. 273, 367-369 (2007).

25. H. I. Bjelkhagen, Silver-Halide Recording Materials: For Holography and Their Processing, Springer Series in Optical Sciences (Springer, 1993), p. 242.

26. T. Rölle, F.-K. Bruder, T. Fäcke, M.-S. Weiser, D. Hönel, and N. Stoeckel, "Photopolymerzusammensetzungen für optische Elemente und visuelle Darstellungen," German patent EP 2172505 A1 (7 April 2010).

27. J. Zhu, G. Wang, Y. Hao, B. Xie, and A. Y. S. Cheng, "Highly sensitive and spatially resolved polyvinyl alcohol/acrylamide photopolymer for real-time holographic applications," Opt. Express 18, 18106-18112 (2010).

28. S. Martin, P. E. Leclere, Y. Renotte, V. Toal, and Y. F. Lion, "Characterization of an acrylamide-based dry photopolymer holographic recording material,"Opt. Eng. 33, 3942-3946 (1994).

29. E. Andrzejewska, "Photopolymerization kinetics of multifunctional monomers," Prog. Polym. Sci. 26, 605-665 (2001).

30. S. Gallego, A. Márquez, D. Méndez, C. Neipp, M. Ortuño, A. Beléndez, E. Fernández, and I. Pascual, "Direct analysis of monomer diffusion times in polyvinyl/acrylamide materials," Appl. Phys. Lett. 92, 073306 (2008).

31. S. Gallego, A. Márquez, D. Méndez, D. C. Neipp, M. Ortuño, M. Álvarez, E. Fernandez, and A. Beléndez, "Real-time interferometric characterization of a polyvinyl alcohol based photopolymer at the zero spatial frequency limit," Appl. Opt. 46, 7506-7512 (2007).

32. P. Hariharan, Optical Holography: Principles, Techniques, and Applications, 2nd ed. Cambridge Studies in Modern Optics (Cambridge University, 1996), p. 47.

33. M. Ortuño, S. Gallego, C. García, I. Pascual, C. Neipp, and A. Beléndez, "Holographic characteristics of an acrylamide/ bisacrylamide photopolymer in $40-1000 \mu \mathrm{m}$ thick layers," Phys. Scr. 2005, 66-68 (2005).

34. C. E. Close, M. R. Gleeson, D. A. Mooney, and J. T. Sheridan, "Monomer diffusion rates in photopolymer material. Part II. High-frequency gratings and bulk diffusion,” J. Opt. Soc. Am. B 28, 842-850 (2011). 
35. S. Gallego, A. Márquez, D. Méndez, S. Marini, A. Beléndez, and I. Pascual, "Spatial-phase-modulation-based study of polyvinyl-alcohol/acrylamide photopolymers in the low spatial frequency range," Appl. Opt. 48, 4403-4413 (2009).

36. S. Gallego, M. Ortuño, I. Pascual, C. Neipp, A. Márquez, and A. Beléndez, "Analysis of second and third diffracted orders in volume diffraction gratings recorded on photopolymers," Phys. Scr. 2005, 58-60 (2005).

37. S. Gallego, A. Marquez, C. Neipp, R. Fernández, F. J. Guardiola, M. Ortuño, I. Pascual, and A. Beléndez, "Model of low spatial frequency diffractive elements recorded in photopolymers during and after recording," Opt. Mater. 38, 46-52 (2014). 